\title{
Relaciones entre Autoconcepto y Bienestar Psicológico en Universitarias Mexicanas
}

\section{Relationships between Self-Concept and Psychological Well-Being in Mexican University Women Students}

\author{
Carolina Jiménez Lira ${ }^{1}$, Jesús Enrique Peinado ${ }^{2}$, Natalia Solano-Pinto ${ }^{3}$, \\ Martha Ornelas ${ }^{4}$ y Humberto Blanco Vega ${ }^{5}$
}

\begin{abstract}
Resumen
Mediante modelos de ecuaciones estructurales se analizan los efectos directos e indirectos del autoconcepto (personal, físico, social y académico) sobre el bienestar psicológico en sus dimensiones de autoaceptación y crecimiento personal. La muestra total fue de 792 universitarias mexicanas, con una edad media de 20.39 años $(\mathrm{DE}=1.81)$. El modelo propuesto obtiene un ajuste satisfactorio. Siendo significativas la mayoría de las relaciones hipotetizadas. El autoconcepto personal y social son las variables con mayor poder explicativo sobre el bienestar psicológico percibido. También destaca la capacidad mediadora del autoconcepto personal entre los factores autoconcepto físico, social y académico y el bienestar psicológico. Futuras investigaciones deberían replicar estos hallazgos en muestras más amplias.
\end{abstract}

Palabras clave: estructuras de covarianza, autoconcepto, bienestar psicológico, ecuaciones estructurales

\begin{abstract}
Through structural equation models analyze the direct and indirect effects of self-concept (personal, physical, social and academic) on psychological well-being in its dimensions of self-acceptance and personal growth. The total sample comprised 792 Mexican university women students, with an average age of 20.39 years $(\mathrm{SD}=1.81$ ). The proposed model obtains a satisfactory fit. Being significant the majority of the hypothesized relationships. Personal and social self-concept are the variables with greater explanatory power over perceived psychological well-being. It also emphasizes the mediator capacity of personal self-concept between physical, social and academic self-concept factors and psychological well-being. Future research should replicate these findings in larger samples.
\end{abstract}

Keywords: covariance structures, self-concept, psychological well-being, structural equation modeling

\footnotetext{
${ }^{1}$ Profesor Investigador. Universidad Autónoma de Chihuahua. Facultad de Ciencias de la Cultura Física. DES Salud. Calle Escorza 900, CP 31000. Chihuahua, Chih. México. Tel.: 6144131507. Correo: cajimenez@uach.mx

${ }^{2}$ Profesor Investigador. Universidad Autónoma de Chihuahua. Facultad de Ciencias de la Cultura Física. DES Salud. Calle Escorza 900, CP 31000. Chihuahua, Chih. México. Tel.: 6144131507. Correo: jpeinado@uach.mx

${ }^{3}$ Profesor Investigador. Universidad de Castilla-La Mancha, España. Facultad de Educación de Toledo. Campus Tecnológico. Avda. Carlos III, 21. 45071 Toledo. Despacho 1.34 Edificio 6. Tel.: 925268800 Ext. 5937. Correo: natalia.solano@uclm.es

${ }^{4}$ Profesor Investigador. Universidad Autónoma de Chihuahua. Facultad de Ciencias de la Cultura Física. DES Salud. Calle Escorza 900, CP 31000. Chihuahua, Chih. México. Tel.: 6144131507. Correo: mornelas@uach.mx

${ }^{5}$ Profesor Investigador. Universidad Autónoma de Chihuahua. Facultad de Ciencias de la Cultura Física. DES Salud. Calle Escorza 900, CP 31000. Chihuahua, Chih. México. Tel.: 6144131507. Correo: hblanco@uach.mx 


\section{Introducción}

El bienestar psicológico se encuentra mencionado en el concepto de salud que ofreció la Organización Mundial de la Salud en su Acta de Constitución en 1948, haciendo referencia a un estado completo de bienestar físico, mental y social y no meramente a la ausencia de enfermedad. Además, el bienestar es una de las prioridades sanitarias mencionadas por la OMS y recogida en la estrategia de Europa 2020. El bienestar, por tanto, se ha considerado como un indicador de calidad de vida. En la actualidad se define al componente cognitivo del bienestar como satisfacción vital; y al componente subjetivo emocional como el bienestar psicológico. Por tanto, se considera al bienestar psicológico como la evaluación del funcionamiento óptimo (Ryan \& Deci, 2001), enfatizando como indicadores de funcionamiento positivo el desarrollo de las potencialidades de la persona y por tanto, el crecimiento personal (Díaz, 2006). Así, por lo tanto, con respecto al bienestar psicológico, una corriente propone que este es el resultado del equilibrio entre las experiencias positivas y negativas que vive un individuo (Lucas, Diener, \& Suh, 1996). Por su parte, Waterman (1993) propone que cuando las personas actúan en congruencia con sus valores e ideales se produce un estado de bienestar psicológico. Uno de los modelos que más ha estudiado el bienestar psicológico es el modelo multidimensional de Ryff (1989). En dicho modelo, el autor integra las perspectivas de la psicología humanista, la psicología evolutiva y la salud mental. Bajo este prisma, punto de vista recogido desde la psicología positiva (Seligman \& Csikszentmihalyi, 2000), el bienestar psicológico implica la estabilidad emocional de una persona a través de una serie de estrategias que le permita vivir con una baja probabilidad de desarrollar problemas mentales (ansiedad, depresión, adicciones) y una alta probabilidad de disfrutar debido a las fortalezas adquiridas. En este sentido, Ryff (2002) considera que el bienestar psicológico debería ser estudiado, entre otras, en relación con desarrollo humano y asociado con otros constructos. En este sentido, el autoconcepto, en concreto alguna de sus dimensiones, ha sido uno de los aspectos estudiados (Rey, Extremera, \& Peña, 2011).

El desarrollo del autoconcepto es un proceso que se inicia en la infancia y se consolida en la etapa de la adultez emergente. Se puede definir como el concepto que una persona tiene de si misma en las distintas facetas vitales. Dicho concepto se considera multidimensional (con aspectos tanto afectivos, congnitivos como conductuales) (Fernández-Zábala, Goñi, Rodríguez- Fernández, \& Goñi, 2015) y se refiere tanto a las cualidades personales como cuánto de competente se siente una persona a la hora de realizar las tareas o exigencias que se requieren en dichas facetas (Shavelson, Hubner, \& Stanton, 1976). Así, la persona adquiere consciencia de su identidad y reconoce sus valores, capacidades, limitaciones; este reconocimiento de los distintos aspectos de su personalidad, le permite desplegar conductas acordes a las situaciones que enfrenta (Fox, 2000). En su modelo, Shavelson et al., (1976) propusieron, como parte del autoconcepto, los apectos académicos y no académicos; específicamente, los no académicos incluyen los dominios de autoconcepto físico, emocional, y social; este modelo ha sido objeto de diversas investigaciones que han ampliado los componentes del autoconcepto (ver Férnandez-Zabala et al., 2015; Goñi, Madariaga, Axpe, \& Goñi, 2011; Goñi Palacios \& Fernández Zabala, 2007). Además, el autoconcepto se consolida en la adultez emergente haciendo que la persona adquiera mayor grado de independencia emocional y social lo que se refleja en el establecimiento de relaciones interpersonales maduras y la creación de objetivos y compromisos desde la responsabilidad (Alvarez, Suárez, Tuero, Núñez, \& Valle, 2015).

Sin embargo, algunos autores señalan la dificultad de sectores de la población juvenil en ejercer el rol que se les presupone en la etapa de adultez emergente (Padovani, 2014). Es decir, no se sienten lo suficientemente competentes para afrontar el papel de adulto e independizarse del ámbito familiar. Así, Andrade (2016) hipotetiza para explicar dichas dificultades, un exceso de apoyo familiar en interacción con la incertidumbre laboral. En este sentido, dicho autor, señala a la población universitaria con mayores competencias $\mathrm{y}$ independencia que cuando se les compara con 


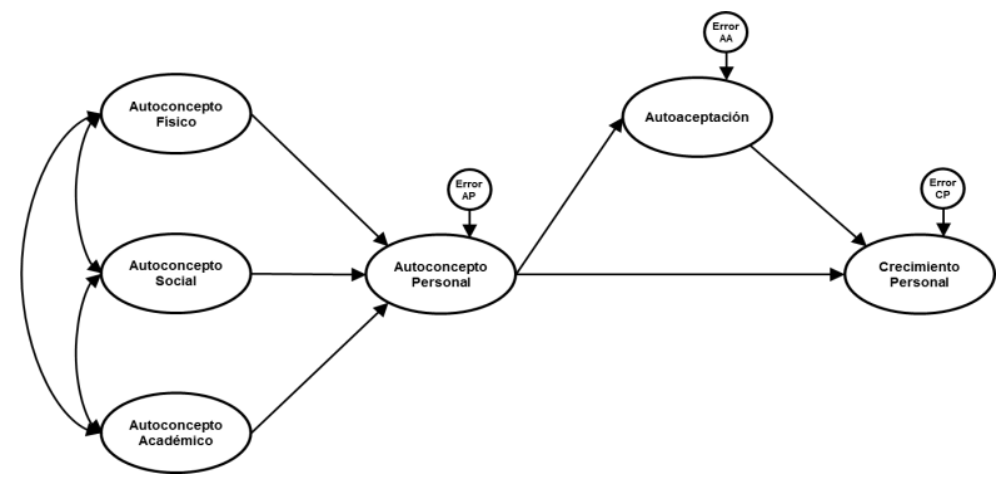

Figura 1. Modelo inicial propuesto para bienestar psicológico en función del autoconcepto. Los indicadores de los factores no están representados por razones de simplicidad en la presentación.

jóvenes inmersos en el mundo laboral. Por el contrario, otros autores postulan que la población universitaria es una población vulnerable porque la demanda de las tareas universitarias requieren un grado de madurez que en ocasiones los estudiantes universitarios no disponen (Bowman, 2010). Además, se considera a las jóvenes mujeres más vulnerables en relación a su salud mental, aunque puntúan más alto en crecimiento personal en pruebas de bienestar psicológico (García-Andalete, 2013). Estos aspectos han justificado el interés de la investigación que se ha hecho patente en el desarrollo de estudios sobre el bienestar psicológico en los estudiantes universitarios. Entre los estudios que han investigado el bienestar psicológico en universitarios destacan los que vinculan a dicha variable con autoconcepto social, físico y personal; de esta forma, Goñi, Rodríguez y Ruiz de Azúa (2004) encuentra relación entre autoconcepto físico y bienestar psicológico; Gámez (2014) establece la relación entre autoconcepto social en la toma de decisiones en estudiantes universitarios lo que les lleva a tener mayor satisfacción en relación a su autonomía personal; Fernández-Zábala, RodríguezFernández y Goñi (2016) señala la necesidad de desarrollar las competencias necesarias para responder a las demandas del funcionamiento social, es decir, el autoconcepto social, y Stallman (2010) enfatiza la importancia de un buen funcionamiento social en el bienestar psicológico.

Por ello los objetivos de este trabajo han sido, en primer lugar, evaluar las propiedades psicométricas del Cuestionario Autoconcepto (AUDIM-M) de Rodríguez y Fernández (2011) y de las escalas crecimiento personal y autoaceptación del cuestionario Escalas de Bienestar Psicológico de
Ryff. En segundo lugar, construir un modelo predictivo de la percepción de bienestar psicológico en las dimensiones autoaceptación y crecimiento personal a partir de los factores autoconcepto personal, social, físico y académico.

\section{Hipótesis}

Del modelo inicial propuesto que integra las relaciones entre bienestar psicológico percibido, y el autoconcepto (Figura 1) se desprenden las hipótesis que a continuación se enuncian.

H1: El factor autoconcepto físico ejerce un efecto indirecto sobre la percepción de bienestar psicológico a través del factor autoconcepto personal.

$\mathrm{H}$ 2: El factor autoconcepto social ejerce un efecto indirecto sobre la percepción de bienestar psicológico a través del factor autoconcepto personal.

H3: El factor autoconcepto académico ejerce un efecto indirecto sobre la percepción de bienestar psicológico a través del factor autoconcepto personal.

H4: El factor autoconcepto personal ejerce un efecto directo sobre la percepción de bienestar psicológico.

H5: La percepción de bienestar psicológico en la dimensión de autoaceptación ejerce un efecto directo sobre la percepción de bienestar psicológico en la dimensión de crecimiento personal.

\section{Método}

\section{Participantes}

Participaron en el estudio 792 mujeres estudiantes universitarias de México. La edad fluctuó entre los 18 y 26 años, con una media de 20.39 y una desviación estándar de 1.81 años. 


\section{Instrumentos}

Escalas de Bienestar Psicológico de Ryff, versión española de Díaz et al. (2006), consta de 39 ítems que se agrupan en 6 dimensiones o subescalas: autoaceptación, relaciones positivas con otras personas, autonomía, dominio del entorno, propósito en la vida, y crecimiento personal. En el modelo estudiado solo se utilizaron las dimensiones crecimiento personal y autoaceptación; eliminando los ítems que mostraron cargas factoriales no aceptables en su dimensión prevista.

Vera-Villarroel, Urzúa, Silva, Pavez y CelisAtenas (2013), analizando los niveles de confiabilidad (consistencia interna y estabilidad temporal) y estructura factorial confirmatoria, en 1646 personas entre 18 y 90 años, encontraron que el modelo teórico de seis dimensiones es el que presenta mejores indicadores de bondad de ajuste para casi todos los grupos de edad evaluados. En cuanto a la evaluación de la estabilidad temporal de la escala, los resultados obtenidos indican que el instrumento es consistente en el tiempo, es decir es confiable, excepto en la dimensión relaciones positivas.

Cuestionario de Autoconcepto (AUDIM) escala tipo Likert de 33 ítems relacionados con la propia persona; donde el encuestado responde, en una escala del 1 a 5 (1=Falso, 2=Más bien falso $3=\mathrm{Ni}$ verdadero ni falso, 4=Más bien verdadero y $5=$ Verdadero) su grado de acuerdo con cada uno de los aspectos propuestos (escogiendo la respuesta que más se ajuste a su persona). Los ítems del cuestionario se agrupan en cuatro factores específicos: autoconcepto académico ( 8 ítems), autoconcepto social (4 ítems), autoconcepto físico (8 ítems) y autoconcepto personal (8 ítems) y autoconcepto general (5 ítems).

En una investigación previa sobre la fiabilidad y validez de este cuestionario (Aguirre, Blanco, Peinado, Mondaca, \& Rangel, 2017), los resultados globales de los análisis factoriales realizados en dos muestras: GFI=.964; RMSEA=.049; CFI=.971 (primera muestra) y GFI=.951; RMSEA=.061; CFI=.952 (segunda muestra) indican un ajuste óptimo para el modelo de medición tetrafactorial (autoconcepto académico, social, físico y personal) del cuestionario AUDIM, donde los cuatro factores obtenidos explican en conjunto aproximadamente el $66 \%$ de la varianza en ambas muestras. Además de que los cuatro factores alcanzaron, en su mayoría, valores de consistencia interna iguales o por encima de .70 , evidenciando una consistencia interna adecuada.

Para este estudio se hicieron tres adaptaciones a la versión original de Rodríguez y Fernández (2011): Primera adaptación, en la versión original se puntúa con cinco opciones de respuesta: (1) falso, (2) más bien falso, (3) ni verdadero ni falso, (4) más bien verdadero y (5) verdadero; en la versión utilizada en la presente investigación el sujeto elige entre 11 posibles respuestas. Conjugamos la escala original con nuestra versión para que quedara de la manera siguiente: falso (0), casi siempre falso (1,2 y 3$)$, a veces verdadero, a veces falso $(4,5$ y 6$)$, casi siempre verdadero $(7,8$ y 9) y verdadero (10). Esta primera adaptación se justifica porque los sujetos al ser estudiantes están acostumbrados a la escala de 0 a 10 , ya que así han sido evaluados por el sistema educativo de México. La segunda adaptación consistió en cambiar algunos términos utilizados en los ítems de la versión original con el fin de utilizar un lenguaje más adecuado al contexto de la cultura mexicana y resumiendo el contenido de $\operatorname{los} 8$ ítems del factor autoconcepto académico en solo 3 ítems (Se me dan bien las asignaturas de gramática y español; Se me dan bien las matemáticas y Soy bueno en las asignaturas de ciencias); motivo por el cual nos referiremos al cuestionario AUDIM como cuestionario AUDIMM. La tercera adaptación consistió en aplicar el instrumento por medio de una computadora; esto con el fin de permitir el almacenamiento de los datos sin etapas previas de codificación, con una mayor precisión y rapidez.

\section{Procedimiento}

Se invitó a participar en el estudio a las alumnas de las licenciaturas que se ofrecen en la Facultad de Ciencias de la Cultura Física (FCCF) de la Universidad Autónoma de Chihuahua. Las que aceptaron participar firmaron la carta de aceptación correspondiente. Luego se aplicaron dos instrumentos de autoreporte: Escalas crecimiento personal y autoaceptación del cuestionario Bienestar Psicológico de Ryff y Cuestionario Autoconcepto (AUDIM-M) por 
medio del ordenador en una sesión de aproximadamente 40 minutos de duración; en los laboratorios o centros de cómputo de la FCCF. Al inicio de la sesión se hizo una pequeña introducción sobre la importancia de la investigación y de cómo acceder a los instrumentos. Se solicitó la máxima sinceridad y se garantizó la confidencialidad de los datos que se obtuvieran. Las instrucciones de cómo responder se encontraban en las primeras pantallas; antes del primer ítem de cada instrumento. Al término de la sesión se les agradeció su participación.

Una vez aplicados los instrumentos se procedió a recopilar los resultados por medio del módulo generador de resultados del editor de escalas versión 2.0 (Blanco et al., 2013).

Por último los resultados obtenidos, se analizaron mediante el paquete SPSS $18.0 \mathrm{y}$ AMOS 21.0.

\section{Análisis de datos}

\section{Análisis de las propiedades psicométricas de los instrumentos}

El primer paso del análisis de las propiedades psicométricas de los cuestionario consistió en calcular la media, la desviaciones estándar, la asimetría, la curtosis y los índices de discriminación de cada ítem. Para luego eliminar aquellos que obtienen una curtosis o asimetría extremas, o un índice de discriminación por debajo de .30 .

Luego, para determinar el número mínimo de factores comunes capaces de reproducir, de un modo satisfactorio, las correlaciones observadas entre los ítems (con buena discriminación) de cada instrumento, se realizó un análisis factorial exploratorio, a partir del método de máxima verosimilitud, tomando como base el criterio de Kaiser-Guttman (Costello \& Osborne, 2005), además de que para garantizar una adecuada representación de las variables (ítems), solo se conservaron aquéllos cuya comunalidad inicial fuera superior a .30 ; después de una rotación varimax (Costello \& Osborne, 2005).

Posteriormente para comprobar la adecuación de la estructura factorial de los instrumentos con la muestra estudiada se realizaron análisis factoriales confirmatorios (AFCs) mediante el programa AMOS 21, las varianzas de los términos de error fueron especificados como parámetros libres, en cada variable latente (factor) se fijó uno de los coeficientes estructurales asociados a uno, para que su escala sea igual a la de una de las variables observables (ítems). El método de estimación empleado fue el de Máxima Verosimilitud; siguiendo la recomendación de Thompson (2004), en el sentido de que cuando se emplea análisis factorial confirmatorio se debe corroborar no sólo el ajuste de un modelo teórico sino que es recomendable comparar los índices de ajuste de varios modelos alternativos para seleccionar el mejor.

Tanto para el Cuestionario Autoconcepto como para las dos escalas de Bienestar Psicológico de Ryff se sometieron a comparación dos modelos de medida: el Modelo 1 (M1), modelo acorde a la distribución original de los ítems dentro de los cuestionarios y el Modelo 2 (M1b), que responde a la estructura factorial conforme a los resultados del análisis factorial exploratorio correspondiente, eliminando los ítems que no fueron suficientemente bien explicados.

Posteriormente, se calculó la fiabilidad de cada una de los factores de los mejores modelos obtenidos en los cuestionarios analizados, a través del Coeficiente Alpha de Cronbach y del Coeficiente Omega.

\section{Análisis de ecuaciones estructurales para el modelo propuesto}

Antes de emplear el análisis de ecuaciones estructurales (SEM) para realizar el análisis del modelo propuesto y poder contrastar las hipótesis planteadas se comprobó que se cumplían los supuestos subyacentes a esta técnica, especialmente los de normalidad y linealidad, para lo cual se analizaron los valores de asimetría y curtosis y los gráficos de dispersión matricial de las distintas variables contempladas en cada modelo.

Luego, a partir de la matriz de correlaciones, se utilizó SEM utilizando el método de estimación de máxima verosimilitud (ML), con la aplicación de procedimientos de remuestreo bootstrap para los casos de no normalidad (Kline, 2011), con el fin de someter a prueba el conjunto de relaciones explicativas hipotetizadas; aun cuando en AMOS 21.0 el ML es especialmente robusto para posibles casos de no normalidad, especialmente si la muestra es suficientemente amplia y los valores 
Tabla 1. Índices absolutos, incrementales y de parsimonia para los modelos generados. Autoconcepto

\begin{tabular}{|c|c|c|c|c|c|c|c|c|c|}
\hline \multirow[b]{2}{*}{ Modelo } & \multicolumn{4}{|c|}{ Índices absolutos } & \multicolumn{3}{|c|}{ Índices incrementales } & \multicolumn{2}{|c|}{ Índices de parsimonia } \\
\hline & $\chi^{2}$ & GFI & RMSEA & SRMR & AGFI & TLI & CFI & CMIN/DF & AIC \\
\hline M1 & $2563.523 *$ & .783 & .091 & .113 & .741 & 684 & .716 & 7.540 & 2695.523 \\
\hline M1b & $85.006^{*}$ & 982 & .041 & .030 & .967 & 978 & 986 & 2.361 & 145.006 \\
\hline
\end{tabular}

Nota. GFI=goodness of fit index; RMSEA=root mean square error of approximation; SRMR=Standardized Root Mean Square Residual; AGFI=adjusted goodness of fit index; TLI=Tucker-Lewis index; CFI= comparative fit index;

$\mathrm{CMIN} / \mathrm{DF}=$ chi-squared fit index divided by degrees of freedom; AIC=Akaike information criterion; ${ }^{*} p<.05$

Tabla 2. Soluciones estandarizadas análisis factorial confirmatorio para el Modelo M1b. Autoconcepto

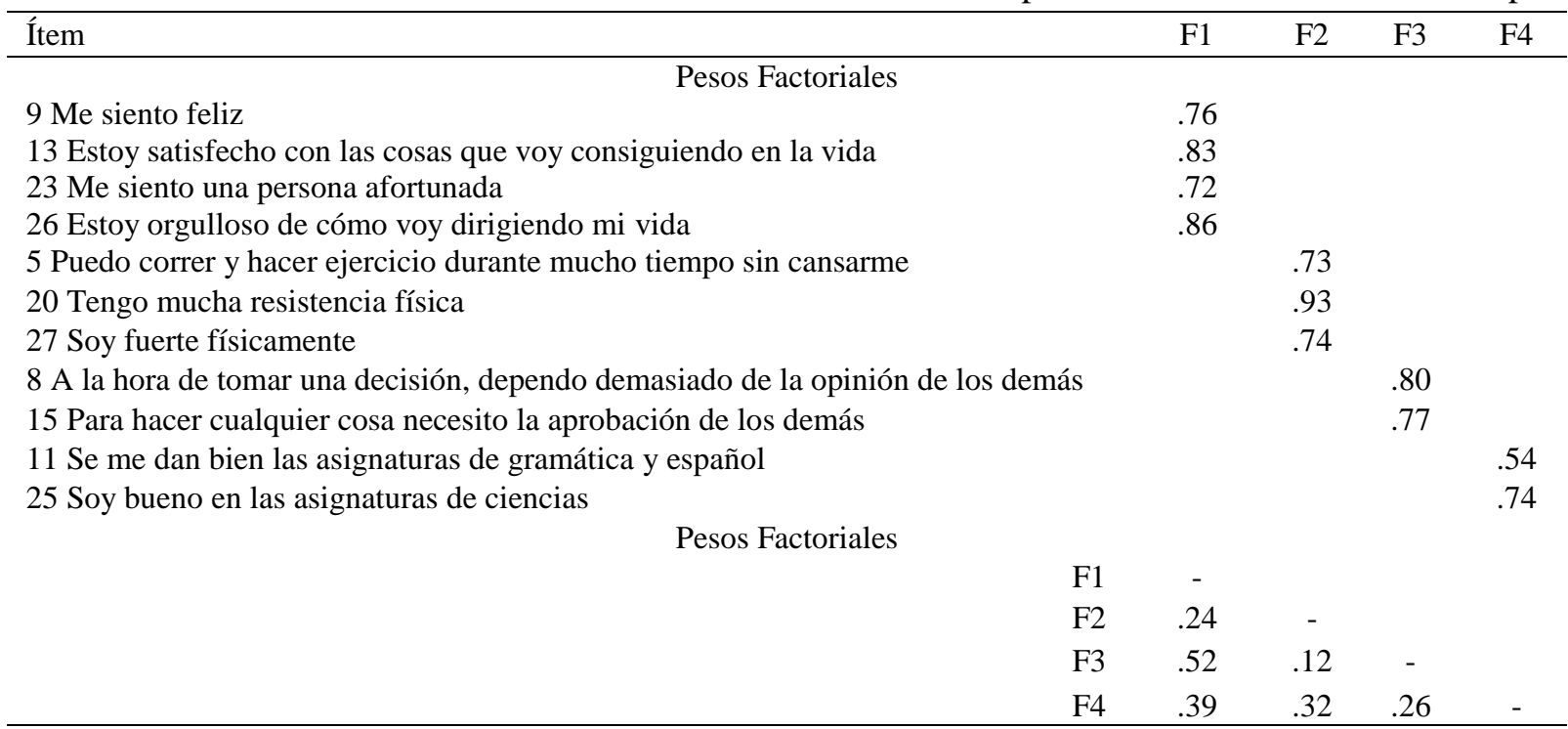

Nota. F1=autoconcepto personal, F2=autoconcepto físico, F3=autoconcepto social, F4=autoconcepto académico

de asimetría y curtosis no son extremos (asimetría $<|2|$ y curtosis $<|7|)$.

El ajuste de los modelos se comprobó a partir del Chi-cuadrado, el índice de bondad de ajuste (GFI), la raíz media cuadrática residual estandarizada (SRMR) y el error cuadrático medio de aproximación (RMSEA) como medidas absolutas de ajuste. El índice de bondad ajustado (AGFI), el Índice Tucker-Lewis (TLI) y el índice de bondad de ajuste comparativo (CFI) como medidas de ajuste incremental. La razón de Chicuadrado sobre los grados de libertad (CMIN/GL) y el Criterio de Información de Akaike (AIC) como medidas de ajuste de parsimonia (Gelabert et al., 2011). Para el GFI, AGFI, TLI y CFI se estableció como umbral de aceptación valores superiores a .90 , e inferiores a .08 para el RMSEA y el SRMR (Gelabert et al., 2011).

Posteriormente, se analizaron los efectos directos, indirectos y totales obtenidos entre las distintas variables contempladas en el modelo.
Análisis de las propiedades psicométricas del Cuestionario Autoconcepto (AUDIM-M)

Los análisis descriptivos y los índices de discriminación (correlación elemento-total corregida) de cada uno de los ítems del cuestionario mostraron que las respuestas a todos los ítems reflejan puntuaciones medias que oscilan entre 4.29 y 9.45, y la desviación estándar ofrece, en todos los casos, valores mayores a 1.30 (dentro de un rango de respuesta entre 0 y 10). Con excepción de los reactivos $2,9,13,23$ y 24 todos los valores de asimetría y curtosis se encuentran dentro del rango \pm 1.50; por lo que se infiere que las variables se ajustan razonablemente a una distribución normal. En cuanto a los índices de discriminación la mayoría de los ítems del cuestionario discriminan satisfactoriamente obteniendo índices de discriminación por encima de .30 .

\section{Análisis factorial exploratorio}

Después de una rotación varimax (Costello \& Osborne, 2005) el análisis factorial exploratorio de los 28 ítems puso de manifiesto una estructura tetrafactorial; llevando a eliminar 9 de los ítems 
Tabla 3. Índices absolutos, incrementales y de parsimonia para los modelos generados. Bienestar Psicológico

\begin{tabular}{|c|c|c|c|c|c|c|c|c|c|}
\hline \multirow[b]{2}{*}{ Modelo } & \multicolumn{4}{|c|}{ Índices absolutos } & \multicolumn{3}{|c|}{ Índices incrementales } & \multicolumn{2}{|c|}{ Índices de parsimonia } \\
\hline & $\chi^{2}$ & GFI & RMSEA & SRMR & AGFI & TLI & $\mathrm{CFI}$ & CMIN/DF & AIC \\
\hline M1 & $609.455^{*}$ & .936 & .078 & .0642 & .908 & .845 & .875 & 9.674 & 665.455 \\
\hline M1b & $47.204 *$ & .989 & .063 & .023 & .967 & .966 & .984 & 6.743 & 75.204 \\
\hline
\end{tabular}

Nota. GFI=goodness of fit index; RMSEA=root mean square error of approximation; SRMR=Standardized Root Mean Square Residual; AGFI=adjusted goodness of fit index; TLI=Tucker-Lewis index; CFI=comparative fit index; CMIN/DF=chi-squared fit index divided by degrees of freedom; AIC=Akaike information criterion ${ }^{*} p<.05$

Tabla 4. Soluciones estandarizadas análisis factorial confirmatorio para el Modelo M1b. Bienestar Psicológico

\begin{tabular}{llc}
\hline Item & F1 & F2 \\
\hline \multicolumn{1}{c}{ Pesos Factoriales } & \\
24 En general, con el tiempo siento que sigo aprendiendo más sobre mí mismo & .70 \\
37 Tengo la sensación de que con el tiempo me he desarrollado mucho como persona & .81 \\
38 Para mí, la vida ha sido un proceso continuo de cambio y crecimiento & .67 & .67 \\
7 En general, me siento seguro y positivo conmigo mismo & .71 \\
19 Me gusta la mayor parte de los aspectos de mi personalidad & .74 \\
31 En su mayor parte, me siento orgulloso de quien soy y la vida que llevo & \\
Correlaciones Factoriales & F1 & - \\
\hline
\end{tabular}

Nota. F1=Crecimiento personal, F2=Autoaceptación

analizados $(1,2,4,7,10,18,21,22$ y 28). El conjunto de los factores seleccionados explicaron aproximadamente el $58 \%$ de la varianza.

\section{Análisis factorial confirmatorio}

Los resultados globales del análisis factorial confirmatorio (GFI .783; RMSEA .091; CFI .716) para el modelo M1 indican que el modelo de medición es no aceptable (Tabla 1).

El conjunto de los cinco factores del modelo M1 explican aproximadamente el 52\% de la varianza.

Por otro lado, solo 9 de los 28 ítems poseen saturaciones iguales o mayores a .70 en su dimensión prevista (ítems 5, 9, 13, 14, 20, 23, 25, 26 y 27). Observándose además intercorrelaciones elevadas entre los factores autoconcepto personal $\mathrm{y}$ autoconcepto general evidenciando una pobre validez discriminante entre ellos.

Los resultados globales del análisis factorial confirmatorio (GFI .982; RMSEA .041; CFI .986), del segundo modelo sometido a prueba (M1b) que corresponde a una estructura tetradimensional del cuestionario acorde a los resultados del análisis factorial exploratorio sin los ítems $(3,6,12,14,16$, 17, 19 y 24) de más baja saturación en cada uno de los factores, indican que este modelo de medición es mejor que el modelo anterior y que su ajuste es óptimo (Tabla 1). Los cuatro factores de este modelo explican en conjunto aproximadamente el 55\% de la varianza. Por otro lado de acuerdo a los resultados de la Tabla 2; con excepción del ítem 11 todos los ítems saturan encima de .70 en su dimensión prevista. Observándose además, intercorrelaciones moderadas entre los factores evidenciando una adecuada validez discriminante entre ellos.

\section{Análisis de las propiedades psicométricas de las dimensiones crecimiento personal $\mathbf{y}$ autoaceptación del Bienestar Psicológico de Ryff}

Los análisis descriptivos y los índices de discriminación (correlación elemento-total corregida) de cada uno de los ítems de las dimensiones crecimiento personal y autoaceptación mostraron que las respuestas a todos los ítems reflejan puntuaciones medias que oscilan entre 2.28 y 4.44, y la desviación estándar ofrece, en todos los casos, valores mayores a .80 (dentro de un rango de respuesta entre 0 y 5). Con excepción de los reactivos 7, 19 y 35 todos los valores de asimetría y curtosis se encuentran dentro del rango \pm 1.50 ; por lo que se infiere que las variables se ajustan razonablemente a una distribución normal. En cuanto a los índices de discriminación la mayoría de los ítems (salvo el 34 y 35) del cuestionario discriminan satisfactoriamente obteniendo índices de discriminación por encima de .35 . 
Tabla 5. Índices absolutos, incrementales y de parsimonia de los modelos inicial y final para bienestar psicológico percibido en función de la actividad física y el autoconcepto físico

\begin{tabular}{|c|c|c|c|c|c|c|c|c|c|}
\hline \multirow[b]{2}{*}{ Modelo } & \multicolumn{4}{|c|}{ Índices absolutos } & \multicolumn{3}{|c|}{ Índices incrementales } & \multicolumn{2}{|c|}{ Índices de parsimonia } \\
\hline & $\chi^{2}$ & GFI & RMSEA & SRMR & AGFI & TLI & CFI & CMIN/DF & AIC \\
\hline Inicial & $314.018^{*}$ & .956 & .048 & .038 & .939 & .955 & .963 & 2.855 & 400.018 \\
\hline Final & $316.157 *$ & .956 & .048 & .039 & .939 & .955 & .963 & 2.848 & 400.157 \\
\hline
\end{tabular}

Nota. GFI=goodness of fit index; RMSEA=root mean square error of approximation; SRMR=Standardized Root Mean Square Residual; AGFI=adjusted goodness of fit index; TLI=Tucker-Lewis index; CFI=comparative fit index; $\mathrm{CMIN} / \mathrm{DF}=$ chi-squared fit index divided by degrees of freedom; AIC=Akaike information criterion; * $p<.05$

\section{Análisis factorial exploratorio}

Después de una rotación varimax (Costello \& Osborne, 2005) el análisis factorial exploratorio de los 13 ítems puso de manifiesto una estructura bifactorial; llevando a eliminar 5 de los ítems analizados $(1,30,34,35$ y 36). El conjunto de los factores seleccionados explicaron el 59\% de la varianza.

\section{Análisis factorial confirmatorio}

Los resultados globales del análisis factorial confirmatorio (GFI .936; RMSEA .078; CFI .875) para el modelo M1 indican que el modelo de medición es no aceptable (Tabla 3).

El conjunto de los dos factores del modelo M1 explican aproximadamente el $43 \%$ de la varianza.

Por otro lado solo 4 de los 13 ítems saturan igual o por encima de .70 en su dimensión prevista (ítems 19, 31, 37 y 38). Observándose además, intercorrelaciones moderadas entre los dos factores evidenciando una adecuada validez discriminante entre ellos.

Los resultados globales del análisis factorial confirmatorio (GFI .989; RMSEA .063; CFI .984), del segundo modelo sometido a prueba (M1b) que corresponde a la estructura bidimensional del Modelo 1, acorde a los resultados del análisis factorial exploratorio, sin los ítems de más baja saturación en cada uno de los factores, indican que este modelo de medición es mejor que el modelo anterior y que su ajuste es óptimo (Tabla 3). Los dos factores de este modelo explican en conjunto aproximadamente el $67 \%$ de la varianza.

Por otro lado de acuerdo a los resultados de la Tabla 4; todos los ítems, salvo los ítems 31 y 38, saturan igual o por encima de .70 en su dimensión prevista. Observándose además, intercorrelaciones moderadas entre los dos factores evidenciando una adecuada validez discriminante entre ellos.

\section{Fiabilidad de los factores obtenidos (consistencia interna)}

Los factores resultantes en los análisis factoriales confirmatorios, en ambos cuestionarios, poseen, en su mayoría, valores de consistencia interna por encima de .70 evidenciando una consistencia interna adecuada para este tipo de subescalas. En concreto tanto para el coeficiente Alpha de Cronbach y el Coeficiente Omega los valores oscilan entre .87 y .76 , excepto para el factor autoconcepto académico donde los valores son .56 y .58 respectivamente.

Modelo de ecuaciones estructurales para predecir el bienestar psicológico a partir del autoconcepto

\section{Evaluación de los supuestos subyacentes al análisis de covarianza}

La mayoría de las variables muestran valores de asimetría de \pm 2.00 y \pm 5.00 de curtosis, además, el índice multivariado de Mardia por encima del valor 70 indica alejamiento de la normalidad multivariada (Rodríguez \& Ruiz, 2008); por lo que se puede inferir que no existe normalidad multivariada.

\section{Ajuste global de los modelos planteados}

Los resultados globales del análisis factorial confirmatorio (GFI .956; RMSEA .048; CFI .963) para el modelo inicial propuesto indican que su ajuste es óptimo (Tabla 5). Sin embargo, dado que una de las relaciones esperadas no resultó significativa (Figura 2) se realizó una reespecificación del modelo inicial eliminando la relación no significativa: autoconcepto personal > crecimiento personal (Figura 3). Tras la eliminación de la vía (path) no significativa, el modelo final GFI .956; RMSEA .048; CFI .963) 


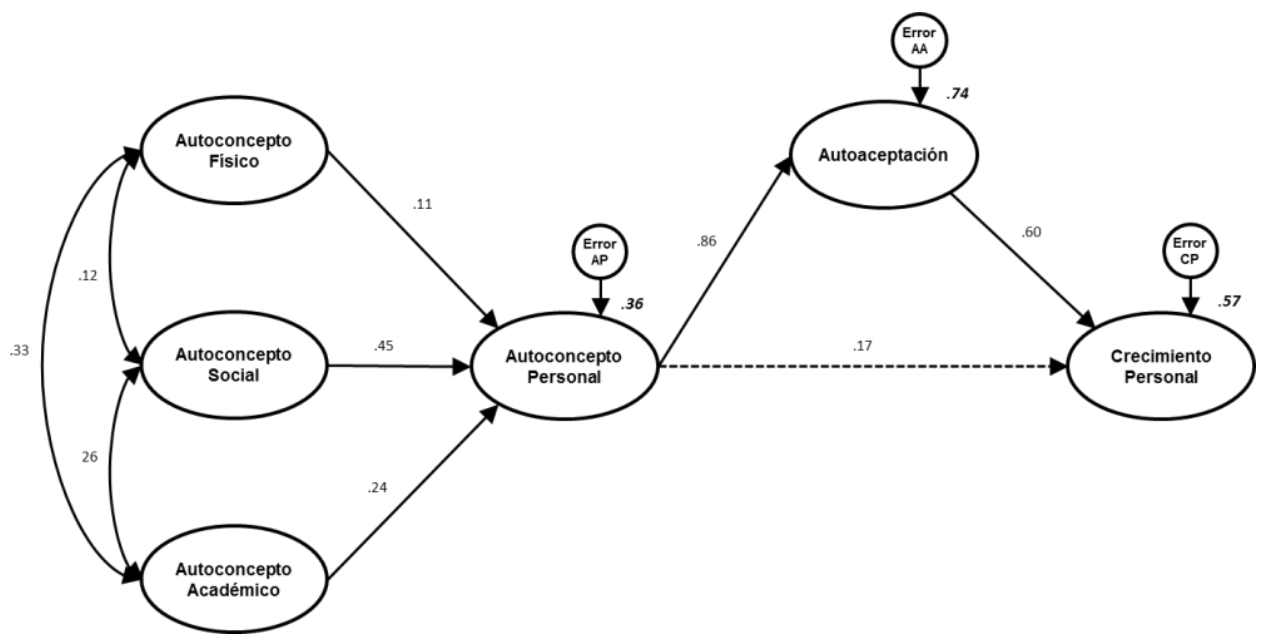

Figura 2. Solución estandarizada del modelo estructural inicial para bienestar psicológico en función del autoconcepto. Todos los parámetros están estandarizados. Las líneas discontinuas representan paths no significativos.

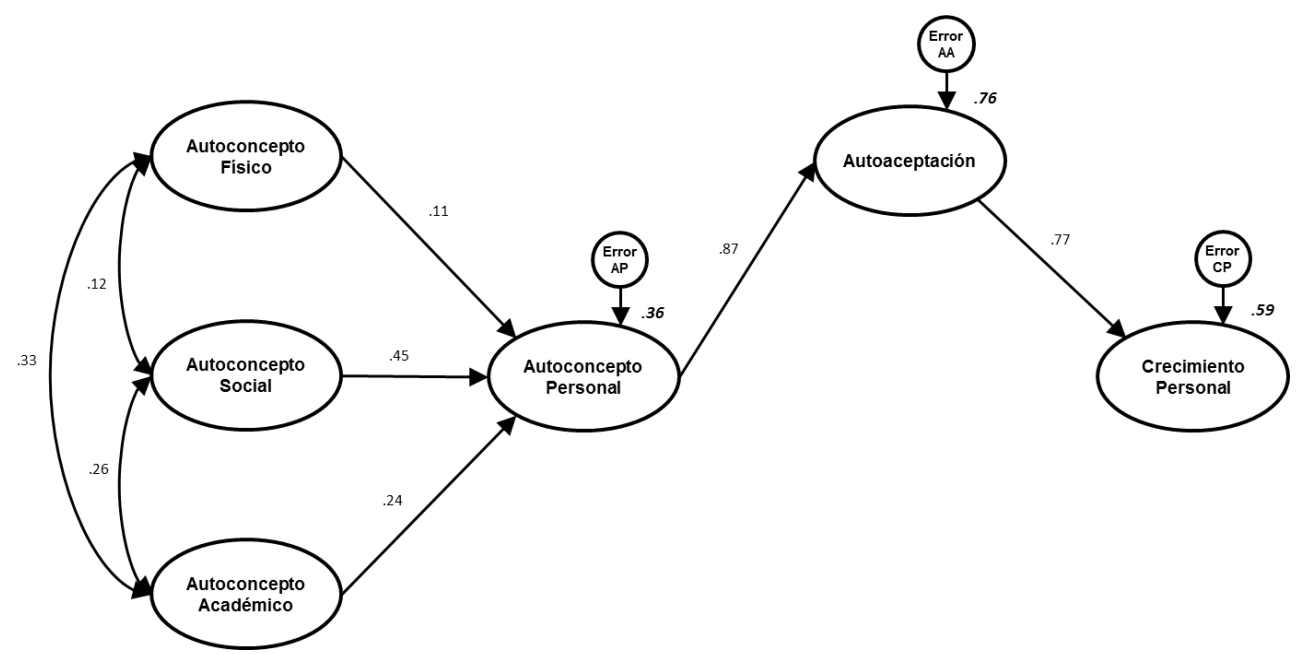

Figura 3. Solución estandarizada del modelo estructural final para bienestar psicológico en función del autoconcepto. Todos los parámetros están estandarizados.

sigue presentando un ajuste óptimo (Tabla 5).

Finalmente, se observa que ambos modelos explican un porcentaje de varianza similar en la variable criterio. El modelo inicial propuesto un $57 \%$ y el modelo final un 59\% de la varianza en la percepción de bienestar psicológico en la dimensión de crecimiento personal (Figura 2 y 3 respectivamente).

\section{Evaluación de los parámetros individuales (modelo final)}

Analizando individualmente los coeficientes de regresión para cada una de las vías propuestas en el modelo final (Figura 3), se observó que la totalidad de las relaciones propuestas obtienen significación cuando menos a un nivel $p<.05$.
El efecto directo más elevado fue el que produce autoconcepto personal en la autoaceptación $(\beta=.87, \quad p<.001)$, seguido del producido por la autoaceptación sobre el crecimiento personal. De manera similar destaca el efecto del autoconcepto social sobre el autoconcepto personal. Finalmente, en la Figura 3 se puede observar que todos los efectos directos son positivos.

Por otro lado, los resultados (Tabla 6) muestran que el autoconcepto social, físico y académico influyen indirectamente de forma significativa en la autoaceptación y el crecimiento personal. Por su parte el autoconcepto personal ejerce un efecto indirecto sobre el crecimiento personal. 
Tabla 6. Efectos directos e indirectos estandarizados entre las variables consideradas en el modelo estructural final para bienestar psicológico percibido en función de la actividad física y el autoconcepto físico

\begin{tabular}{lcccccc}
\hline & & $\begin{array}{c}\text { Autoconcepto } \\
\text { Social }\end{array}$ & $\begin{array}{c}\text { Autoconcepto } \\
\text { Físico }\end{array}$ & $\begin{array}{c}\text { Autoconcepto } \\
\text { Académico }\end{array}$ & $\begin{array}{c}\text { Autoconcepto } \\
\text { Personal }\end{array}$ & $\begin{array}{c}\text { Crecimiento } \\
\text { Personal }\end{array}$ \\
\hline $\begin{array}{l}\text { Autoconcepto } \\
\text { Personal }\end{array}$ & $\begin{array}{c}\text { Directo } \\
\text { Indirecto } \\
\text { Directo }\end{array}$ & .450 & .114 & .244 & & \\
$\begin{array}{l}\text { Autoaceptación } \\
\text { Crecimiento }\end{array}$ & $\begin{array}{c}\text { Indirecto } \\
\text { Directo } \\
\text { Personal }\end{array}$ & .392 & .100 & .212 & & .870 \\
\hline
\end{tabular}

\section{Conclusiones y Discusión}

Los análisis factoriales exploratorios y confirmatorios llevados a cabo pusieron de manifiesto una estructura tetrafactorial: autoconcepto personal, autoconcepto físico, autoconcepto social y autoconcepto académico para el cuestionario de autoconcepto (AUDIM-M) sin embargo el modelo obtenido no coincide con el planteado por Rodríguez y Fernández (2011), ya que para logra un mejor ajuste hubo que eliminar un factor varios de los ítems analizados. Mientras que para las escalas crecimiento personal y autoaceptación del cuestionario de Bienestar Psicológico de Ryff muestran que una estructura dos factores coincidiendo, salvo por la eliminación de varios de ítems, con la versión española propuesta por Díaz et al. (2006).

Con respecto a la predicción del bienestar psicológico percibido a través del autoconcepto se han cumplido la mayoría de las hipótesis planteadas a partir del modelo inicial propuesto, de tal modo que los factores autoconcepto personal, físico, social y académico predicen positivamente la percepción de bienestar psicológico en la dimensión de autoaceptación y ésta a su vez ejerce un efecto directo positivo sobre la percepción de bienestar psicológico en la dimensión de crecimiento personal. En concreto, autoconcepto personal como factor de autoconcepto es el factor de predicción positivo directo de la dimensión de autoaceptación de bienestar psicológico. Este resultado se puede explicar por la similitud del contenido de los ítems de ambos factores, por ejemplo, estoy orgulloso de cómo voy dirigiendo mi vida (ítem de autoconcepto personal) y me siento orgulloso de quién soy y la vida que llevo (ítem de autoaceptación-dimensión de bienestar psicológico). En particular los factores autoconcepto físico, social y académico ejercen un efecto indirecto sobre la percepción de bienestar psicológico a través del factor autoconcepto personal y este, a su vez, produce un efecto directo sobre el bienestar psicológico. En parte, estos resultados coinciden en parte en los estudios que enfatizan la importancia del autoconcepto físico y el bienestar psicológico (Goñi, et al, 2014); los que resaltan la importancia del autoconcepto social en la autonomía personal (Fernández-Zábala, et al., 2016; Stallman, 2010). Sin embargo, en los estudios revisados no se establece la relaciones entre las dimensiones del autoconcepto en su carácter predictivo hacia el bienestar psicológico, lo que estimamos tiene un valor novedoso en los hallazgos encontrados. Las variables incluidas en el modelo constituyen un entramado particular de relaciones de modo que ninguno de estos constructos funciona independientemente, sino que se interrelacionan, llegando casi a solaparse unos sobre otros.

El conjunto de variables consideradas en el estudio explican, en buena medida, el bienestar psicológico percibido de los alumnos universitarios estudiados y constituyen, de este modo, un ámbito necesario de intervención educativa. Así, las variables contempladas en el modelo alcanzan a explicar más del $50 \%$ de la varianza total en el bienestar psicológico percibido, lo que parecen revelar la importancia del conjunto de variables estudiadas en la predicción/explicación del bienestar psicológico de los universitarios $\mathrm{y}$, por consiguiente, la necesidad de incluir su optimización y desarrollo en los currículos escolares. Así, se considera que la población universitaria es una población especialmente relevante para el futuro de la sociedad. De esta forma, se recomienda la protección de esta población insertando medidas que promuevan las fortalezas psicológicas para que aumente el bienestar psicológico. Tal y como se señala por algunos autores, no hay posibilidad 
de progreso en los países desarrollados si no se cuida la salud mental (Bloom, et al., 2011).

\section{Referencias}

Aguirre, J. F., Blanco, H., Peinado, J. E., Mondaca, F., \& Rangel, Y. S. (2017). Factorial composition of the Dimensional Self-Concept Questionnaire AUDIM-M in Mexican university students. Revista Electrónica Nova Scientia, 9(18), 627-645 doi:10.21640/ns.v9i18.830

Alvarez, A., Suárez, N., Tuero, E., Núñez, J.C., Valle, A., \& Regueiro, B. (2015). Implicación familiar, autoconcepto del adolescente y rendimiento académico. European Journal of Investigation Health, Psychology and Education, 5(3), 293-311 doi:10.30552/ejihpe.v5i3.133

Andrade, C. (2016). A construção da Identidade, auto-conceito e autonomía em Adultos Emergentes. Psicologia Escolar $e$ Educacional, 20(1), 137-146 http://dx.doi.org/10.1590/21753539/2015/0201944

Blanco, H., Ornelas, M., Tristán, J. L., Cocca, A., Mayorga-Vega, D., López-Walle, J., \& Viciana, J. (2013). Editor for creating and applying computerise surveys. Procedia Social and Behavioral Sciences, 106, 935-940 doi:10.1016/j.sbspro.2013.12.105

Bloom, D. E., Cafiero, E. I., Jané- Llopis, E., Abrahams- Gessel, S., Bloom, L. R., Fathima, S., y Weinstein, C. (2011). The global economic burden of non-communicable diseases. Ginebra: Wordl Economic Forum.

Costello, A. B., \& Osborne, J. W. (2005). Best practices in exploratory factor analysis: Four recommendations for getting the most from your analysis. Practical Assessment, Research and Evaluation, 10(7), 1-9.

Díaz, D., Rodríguez-Carvajal, R., Blanco, A., Moreno-Jiménez, B., Gallardo, I., Valle, C., \& Dierendonck, D. V. (2006). Adaptación española de las escalas de bienestar psicológico de Ryff. Psicothema, 18(3), 572577.

Fernández- Zábala, A., Goñi, E., RodríguezFernández, A., \& Goñi, A. (2015). Un nuevo cuestionario en castellano con escalas de las dimensiones del autoconcepto. Revista Mexicana de Psicología, 32(2), 149-159 doi:10.6018/analesps.32.1.193931

Fernández- Zábala, A., Rodríguez- Fernández, A., \& Goñi, A. (2016). The structure of the Social Self-Concept Questionnaire. Anales de Psicología, 32(1), 199-205. http://dx.doi.org/10.6018/analesps.32.1.193931

Fox, K. (2000). Self-esteem, self perception, and exercise. International Journal of Sport Psychology, 31, 228-240.

Gámez, E., Díaz, J. M., Marrero, H., Galindo, Mª P., \& Breva, A. (2014). Relaciones entre el autoconcepto relacional, la elección de metas y la satisfacción de necesidades psicológicas en estudiantes universitarios. Universitas Psychologia, 13(4), 1289-1303 doi:10.11144/Javeriana.UPSY13-4.rarm

García- Andalete, J. (2013). Bienestar psicológico, edad y género en universitarios españoles. Salud y Sociedad, 14(1), 48-58 doi:10.22199/S07187475.2013.0001.00004

Gelabert, E., García-Esteve, L., Martín-Santos, R., Gutiérrez, F., Torres, A., \& Subirà, S. (2011). Psychometric properties of the Spanish version of the Frost Multidimensional Perfectionism Scale in women. Psicothema, 23(1), 133-139.

Goñi, E., Madariaga, J. M., Axpe, I., \& Goñi, A. (2011). Structure of the personal self-concept (PSC) questionnaire. International Journal of Psychology and Psychological Therapy, 11(3), 509-522.

Goñi, E., \& Fernández, A. (2007). Los dominios social y personal del autoconcepto. Revista de Psicodidáctica, 12(2), 179-194. doi:10.1387/RevPsicodidact.218

Goñi, A., Rodríguez, A., \& Ruiz de Azúa, S. (2004). Bienestar psicológico y autoconcepto físico en la adolescencia y juventud. Psiquis, 25(4), 141-151.

Keyes, C.L., Shmotkin, D., \& Ryff, C.D. (2002). Optimizing well-being: The empirical encounter of two traditions. Journal of Personality and Social Psychology, 82(6), 719-727 doi:10.1037//0022-3514.69.4.719

Kline, R. B. (2011). Principles and practice of structural equation modeling. New York: Guilford Press. 
Lucas, R. E., Diener, E., \& Suh, E. M. (1996). Discriminant validity of well-being measures. Journal of Personality and Social Psychology, 71(3), 616-628 doi:10.1037/0022-3514.71.3.616

Padovari, R. C., Neufeld, C. B., Maltoni, J., Barbosa, L. N F., Souz, W. F., Cavalcanti, H. A. F., \& Lameu, J. N. (2014). Vulnerabilidade e bem-estar psicológicos do estudante universitário. Revista Brasileira de Terapias Cognitivas, 10(1), 2-10 doi:10.5935/1808-5687.20140002

Rey, L., Extremera, N., \& Peña, M. (2011). Perceived emotional intelligence, self- esteem and life satisfaction in adolescents. Psychosocial Intervention, 20(2), 227-234. doi:10.5093/in2011v20n2a10

Ryan, R. M., \& Deci, E. L. (2001). On happiness and humor potentials: A review of research on hedonic and audaimonic well-being. Annual Review of Psychology, 52(1), 141-166 doi:10.1146/annurev.psych.52.1.141

Ryff, C. D. (1989). Happiness is everything, or is it? Exploration on the meaning of psychological well-being. Journal of Personality and Social Psychology, 57(6), 1069-1081 doi:10.1037/0022-3514.57.6.1069

Rodríguez, A., \& Fernández, A. (2011). Propiedades psicométricas del Cuestionario de Autoconcepto Dimensional AUDIM. In J. M. Román, M. Á. Carbonero y J. D. Valdivieso (Eds.), Educación, aprendizaje y desarrollo en una sociedad multicultural (pp. 999-1113). Madrid: Ediciones de la Asociación Nacional de Psicología y Educación.

Rodríguez, M. N., \& Ruiz, M. A. (2008). Atenuación de la asimetría y de la curtosis de las puntuaciones observadas mediante transformaciones de variables: Incidencia sobre la estructura factorial. Psicológica, 29(2), 205-227.

Seligman, M. E. P., \& Csikszentmihalyi, M. (2000). Positive psychology. An introduction. American Psychologist, 55, 5-14. Edición Especial. doi:10.1037/0003-066X.55.1.5

Shavelson, R. J., Hubner, J. J., \& Stanton, J.C. (1976). Self concept validation of construct interpretations. Review of Educational Research, 46(3), 407-441 doi:10.3102/00346543046003407
Stallman, H.M. (2010). Psychological distress in university students: A comparison with general population data. Australian Psychologist, 45(4), 249-557. doi:10.1080/00050067.2010.482109

Thompson, B. (2004). Exploratory and confirmatory factor analysis. Understanding concepts and applications. Washington, D C: American Psychological Association doi:10.1037/10694-000

Vera-Villarroel, P., Urzúa, A., Silva, J. R., Pavez, P., \& Celis-Atenas, K. (2013). Escala de Bienestar de Ryff: Análisis comparativo de los modelos teóricos en distintos grupos de edad. Psicologia: Reflexão e Crítica, 26(1), 106112 doi:10.1590/S0102-79722013000100012

Waterman, A. S. (1993). Two conceptions of happiness: Constrasts of personal expressiveness (eudaimonia) and hedonic enjoyment. Journal of Personality and Social Psychology, 54(6), 1063-1070 doi:10.1037/0022-3514.64.4.678

Word Health Organization (1948). Constitution of the Word Health Organization. Recuperado de http: //www.saro.who.int/LinkFiles/ABOUT_SEA RO_const.pdf

Word Health Organization (2013). The European health report 2012: Charting the way to wellbeing. Copenhague: WHO. Recuperado de http://www.euro.who.int/_data/assets/pdf_fil e/0004/197113/EHR2012-Eng.pdf 